\title{
An Outbreak of NCIP (2019-nCoV) Infection in China - Wuhan, Hubei Province, 2019-2020
}

\author{
The 2019-nCoV Outbreak Joint Field Epidemiology Investigation Team ${ }^{1,2,3}$; Qun Li ${ }^{1, *}$
}

Emerging and re-emerging pathogens are great challenges to the public health (1). A cluster of cases of viral pneumonia of unknown etiology (VPUE), now known as novel coronavirus-infected pneumonia (NCIP), occurred in Wuhan, Hubei Province and was reported to health authorities on December 29, 2019 (2). A national, provincial, and municipal joint investigation team has been assembled to conduct field investigations and implement disease control and prevention measures. This report shows interim results of the investigation and makes recommendations for response measures.

\section{Identification of the Epidemic}

On December 29, 2019, a hospital in Wuhan admitted four individuals with pneumonia and recognized that all four had worked in the Huanan Seafood Wholesale Market, which sells live poultry, aquatic products, and several kinds of wild animals to the public. The hospital reported this occurrence to the local center for disease control (CDC), which lead Wuhan CDC staff to initiate a field investigation with a retrospective search for pneumonia patients potentially linked to the market. The investigators found additional patients linked to the market, and on December 30, health authorities from Hubei Province reported this cluster to China CDC. The following day, China CDC sent experts to Wuhan to support the investigation and control effort. Samples from these patients were obtained for laboratory analyses.

To identify potential cases, a temporary surveillance case definition for the VPUE was established at that time that was consistent with previous "pneumonia of unknown etiology" definitions: an illness of unknown etiology with 1) fever with or without a recorded temperature; 2) radiographic evidence of pneumonia; 3) low or normal leukocyte count or low lymphocyte count during the early stage of disease; and 4) no improvement or worsening symptoms after 3 to 5 days of antimicrobial treatment per standard clinical guidelines (3).

A probable case of VPUE was defined as a surveillance VPUE case or an illness of unknown etiology fulfilling the first three surveillance VPUE case criteria with a history of exposure to the Huanan Seafood Wholesale Market in Wuhan or any other VPUE case.

The initial laboratory screening results from patients were negative for 26 common respiratory pathogens, including influenza $\mathrm{A}$ and $\mathrm{B}$ virus, parainfluenza virus, adenovirus, respiratory syncytial virus, metapneumovirus, rhinovirus, enterovirus, and other common respiratory viruses. On January 3, 2020, the sequence of novel $\beta$-genus coronaviruses $(2019-\mathrm{nCoV})$ was determined from specimens collected from patients in Wuhan by scientists of the National Institute of Viral Disease Control and Prevention (IVDC), and three distinct strains have been established (2). On January 7 , this novel coronavirus was confirmed to be the pathogenic cause of this VPUE cluster, and the disease has been designated NCIP.

\section{Epidemiological Findings}

Patients' clinical manifestations were consistent with viral pneumonia. Most patients had severe and nonproductive cough following illness onset, some had dyspnea, and almost all had normal or decreased leukocyte counts and radiographic evidence of pneumonia.

Huanan Seafood Wholesale Market has western and eastern sections, and 15 environmental specimens collected in the western section were positive for 2019$\mathrm{nCoV}$ virus through $\mathrm{RT}$-PCR testing and genetic sequencing analysis. Despite extensive searching, no animal from the market has thus far been identified as a possible source of infection.

As of January 19, 198 cases of NCIP have been reported in Wuhan with $110(55.6 \%)$ cases reported in males. The median age was 57 years old with a range of 26 to 89 years. As of this day, 25 patients have 
recovered and have been discharged from medical care, and 3 patients have died. A total of 170 patients are receiving isolated medical treatment at designated Wuhan medical facilities. Among these patients, 126 are classified to have mild illnesses, 35 have severe illnesses, and 9 are in critical condition. A total of 817 close contacts of cases have been placed under quarantine for medical observation, of which 727 have been cleared and 90 remain in quarantine. No cases of NCIP have been found among the close contacts.

A total of 16 health care workers (HCWs) are believed to have been infected while caring for patients from the outbreak; 15 have confirmed NCIP, and 1 $\mathrm{HCW}$ is still under being verified.

Two clusters containing two cases and three cases, respectively, were identified among the confirmed cases. Human-to-human transmission appears to have occurred in the two-person cluster.

The epidemiological investigation of 198 confirmed cases revealed that $22 \%$ of patients had direct exposure to the Huanan Seafood Wholesale Market before illness onset; $32 \%$ of patients had contact with patients with fever or respiratory symptoms; and $51 \%$ of cases had neither visited the Huanan Seafood Wholesale Market nor had contact with similar patients before their illness onset.

\section{Preliminary Conclusions}

This outbreak was detected in Wuhan in late December 2019 and was caused by a novel coronavirus 2019-nCoV. Although investigation into the source or sources of NCIP is ongoing, exported cases have been reported by Thailand, Japan, Republic of Korea, and other provinces in China, indicating that the epidemic is ongoing in Wuhan and that further spread is almost certain.

During the investigation, the following was concluded: 1) recently-reported, confirmed cases have had no history of exposure to the Huanan Seafood Wholesale Market; 2) human-to-human transmission has occurred; and 3) HCWs have been shown to have been infected by the novel coronavirus. Based on this information, transmission within communities is believed to be taking place in Wuhan.

Measures to prevent or reduce transmission should be implemented, including use of personal protective equipment (surgical masks), school closures, postponement or cancellation of mass gatherings, and exit screenings.

\# Corresponding author: Qun Li, liqun@chinacdc.cn.

${ }^{1}$ Chinese Center for Disease Control and Prevention, Beijing, China ${ }^{2}$ Hubei Provincial Center for Disease Control and Prevention, Wuhan, Hubei, China; ${ }^{3}$ Wuhan Municipal Center for Disease Control and Prevention, Wuhan, Hubei, China.

Submitted: January 20, 2020; Accepted: January 21, 2020

\section{References}

1. George F. Gao. From “A” IV to "Z” IKV: attacks from emerging and Reemerging pathogens. Cell 2018;172(6):1157 - 9. http://dx.doi.org/10. 1016/j.cell.2018.02.025.

2. Tan WJ, Zhao X, Ma XJ, Wang WL, Niu PH, Xu WB, et al. A novel coronavirus genome identified in a cluster of pneumonia cases Wuhan, China 2019-2020. China CDC Weekly 2020;2(4):61 -2. http://weekly.chinacdc.cn/en/article/ccdcw/2020/4/61.

3. Ministry of health protocol of surveillance, investigation and management for pneumonia of unknown etiology, 2007. Ministry of Health, China. 